\title{
Influence of Counterions on the Helical Conformations of Charged Polypeptide Chains
}

The circular dichroism spectra of polypeptides with charged side chains indicate that the main chain assumes a local conformation which is regular rather than random.1,2 Calculations of the minimum energy conformations which result from the repulsive interactions between these charges show that this structure should consist of short sequences of an extended left-handed helix with approximately 2.5 peptide residues per turn. ${ }^{3}$ We now explicitly consider the effect which counterions would be expected to have on such chain conformations.

In order to extend the previous calculations ${ }^{3}$ we have now included counterion as well as side-chain charges. A charge of equal magnitude and opposite sign was placed at a distance $\Delta r$ from the side-chain charge on the extension of the $\mathrm{C}^{\alpha}-\mathrm{C}^{\beta}$ line and the total electrostatic energy computed as a function of $\phi$ and $\psi$. (This location of the counterion clearly corresponds to minimum energy as $\Delta r \rightarrow 0$. We also find such an eclipsed arrangement to be of lower energy than a staggered arrangement for the case of two concentric circles with equal numbers of opposite charges, except when $\Delta r$ is very large, in which case, as we shall see, the effect of the counterions is negligible. While a complete minimization of energy has not been done for the helix, other than for the above radial location of the counterions, we believe that, by analogy with the circular model, this arrangement will be close to if not that of lowest energy, and that in any case the qualitative features of our model will be correct.) The dielectric constant of the chain $\epsilon$ was taken to be 10 , as in the earlier calculations, ${ }^{3}$ and the dielectric constant of the medium between the side-chain charge and the counterion $\epsilon^{\prime}$ was varied. The computations were done for a chain of 16 residues.

Some of the results of this calculation are shown in Tables I and II. In Table I the position and energy of the minimum in the $\phi, \psi$ plot for the left-handed helix are given as a function of $\Delta r$ for $\epsilon^{\prime}=30$. It will be seen that the position of the minimum is essentially the same as for the case of no counterions, and that the energy at this minimum is always repulsive and large (although of course much smaller than the very large repulsive energy $(\sim 23 \mathrm{kcal} /$ mole for $\Delta r=10 \AA$ and $\sim 19 \mathrm{kcal} /$ mole for $\Delta r=2 \AA)$ associated with, for example, the $\alpha$-helix conformations). In Table II the same quantities are given as a function of $\epsilon^{\prime}$ for $\Delta r=5 \AA$. An interesting behavior is obtained in this case. As $\epsilon^{\prime}$ decreases the position of the minimum does not change substantially, but the energy at this minimum decreases rapidly. When $\epsilon^{\prime}=10$ much of the $\phi, \psi$ map is characterized by energies close to zero, with some points being associated with small attractive energies (which is not surprising since the repulsive energies are being increasingly counter-balanced by attractive interactions). 'The lowest energy position, i.e., $\phi=85^{\circ}$ and $\psi=95^{\circ}$, is given in Table $\mathrm{II}$, and this region remains so for increasingly attractive counterion configurations: for example, for $\epsilon^{\prime}=10$ and $\Delta r=1 \AA$ (the latter, of course, being not too physically reasonable) we find that $\phi=100^{\circ}, \psi=100^{\circ}$, and $E=-5.44 \mathrm{kcal} /$ mole at the minimum. Since the electrostatic energy is now close to zero over most of the map, we expect the steric energy to be predominant in determining the conformation. Thus, with the $\alpha$-helix having a steric energy of about $-8 \mathrm{kcal} /$ mole, ${ }^{4}$ the favored conformation will be the $\alpha$-helix. (The steric energy near $\phi=100^{\circ}$ and $\psi=100^{\circ}$ is very high, and thus this conformation will not be favored even though it corresponds to an attractive coulombic interaction.)

We may picture the effect of counterions on the helical structure of charged polypeptide chains therefore as follows. In media of high dielectric constant (e.g., water) and in the absence of excess counterions, the repulsive interactions between side-chain 
TABLE I

Coulombic Energy Minimum for PLGA $(N=16)$ as a Function of $\Delta r$ $\left(\epsilon=10, \epsilon^{\prime}=30\right)$

\begin{tabular}{rrrc}
$\Delta r, \AA$ & $\phi_{\text {min }}$ & $\psi_{\text {min }}$ & $E_{\text {min }}, \mathrm{kcal} / \mathrm{mole}$ \\
\hline$-\mathrm{a}$ & 45 & -5 & 15.82 \\
10.0 & 50 & -5 & 11.70 \\
8.0 & 50 & -5 & 11.43 \\
6.0 & 50 & -5 & 11.15 \\
4.0 & 50 & -5 & 10.88 \\
2.0 & 45 & -5 & 10.65 \\
1.0 & 45 & -5 & 10.58 \\
\hline
\end{tabular}

a Corresponds to the case of no counterions.

TABLE II

Coulombic Energy Minimum for PLGA $(N=16)$ as a Function of $\epsilon^{\prime}$ $(\epsilon=10, \Delta r=5 \AA)$

\begin{tabular}{ccrc}
\hline$\epsilon^{\prime}$ & $\phi_{\min }$ & $\psi_{\min }$ & $E_{\min }, \mathrm{kcal} / \mathrm{mole}$ \\
\hline 30 & 50 & -5 & 11.01 \\
25 & 50 & -5 & 10.05 \\
20 & 45 & 0 & 8.60 \\
15 & 45 & 0 & 6.19 \\
10 & 85 & 95 & -0.19 \\
\hline
\end{tabular}

charges predominate, and the polypeptide backbone assumes an extended-helix conformation. If the dielectric constant is decreased, we expect a destabilization of the extended-helix structure, both because the counterions are attracted closer to the sidechain charges and because the attractive energy increases. When the dielectric constant is low enough so that attractive energies counterbalance repulsive energies to the extent that steric energies predominate, we would expect the system to transform completely to the $\alpha$-helix conformation. If the counterion concentration is increased, the attractive interactions would increase (because of a smaller $\Delta r$ or because the counterion "sites" are occupied for a greater fraction of time) and the extended-helix structure would again be destabilized.

Experimental evidence confirms the above predicted behavior. Poly (L-glutamic acid) in aqueous solution at $\mathrm{pH} 7$ (where it is charged) transforms from the extended helix to the $\alpha$-helix upon addition of methanol, the transition being relatively sharp." A similar effect is observed for charged poly-L-lysine..$^{5}$ In the latter case, the transition occurs at $87-90 \%$ methanol but at $76 \%$ isopropanol, and it is interesting to note that these solvent mixtures have similar dielectric constants (about 38 and 33 , respectively), although the dielectric constants of the pure alcohols are significantly different (32.6 for methanol and 18.3 for isopropanol). This would be expected on the basis of our proposed model. The influence of excess counterions as well as change in dielectric constant of the solvent are illustrated in Figure 1. The upper curve shows the circular dichroism spectrum of poly (L-glutamic acid) in water at $\mathrm{pH} 7$ or in $33 \%$ methanol (the curves are essentially the same), the positive band near $220 \mathrm{~m} \mu$ indicating an extended-helix structure. ${ }^{1}$ If the water solution is made $1.8 \mathrm{M}$ in $\mathrm{LiCl}$, the extended-helix structural component diminishes. For a solution which is $1.8 \mathrm{M}$ in $\mathrm{LiCl}$ and $30 \%$ in methanol, the 


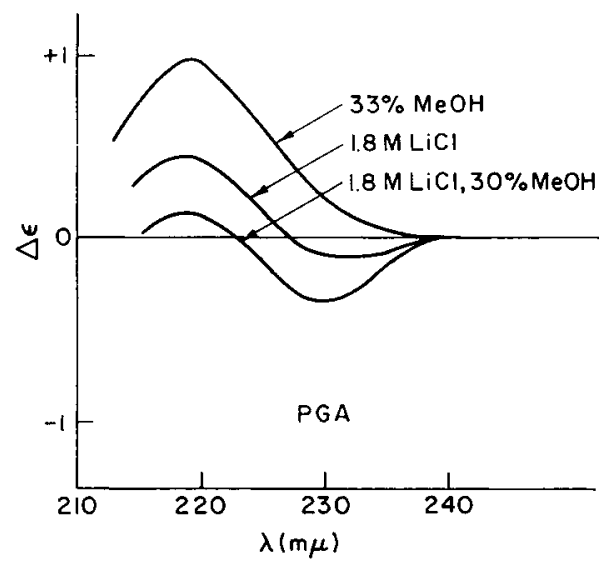

Fig. 1. Circular dichroism spectra of poly(L-glutamic acid): upper curve-in water at $\mathrm{pH} 7$ or in $33 \%$ methanol (curves are almost the same); middle curve-in aqueous $1.8 \mathrm{M} \mathrm{LiCl}$; lower curve-in $1.8 \mathrm{M} \mathrm{LiCl}$ and $30 \%$ methanol.

extended-helix component decreases further. Both of these features are consistent with the predictions of the model. Finally, it might be noted that increasing temperature decreases the $\alpha$-helix content of poly-L-lysine, more so at $88 \%$ methanol than at $98 \%$ methanol. ${ }^{5}$ This can again be understood on the basis of our model if we make the (not unreasonable) assumption that $\overline{\Delta r}$ for the counterions increases with temperature, thus diminishing the attractive coulombic interactions with respect to the repulsive and therefore destabilizing the $\alpha$-helix relative to the extended helix.

We see therefore that the conformation of a charged polypeptide chain is determined by the relative magnitudes of coulombic and steric energies. The former depends explicitly on counterion concentration and is particularly sensitive to the dielectric constant of the solvent. The incorporation of these factors permits an understanding of the dependence of the transition between $\alpha$-helix and extended-helix structures on alcohol content and also explains the effect of added salt.

This research was supported by U.S. Public Health Service grant AM 02830 (SK) and National Science Foundation grant GP-7365 (JEM).

\section{References}

1. M. L. Tiffany and S. Krimm, Biopolymers, 6, 1379 (1968).

2. M. L. Tiffany and S. Krimm, Biopolymers, in press.

3. S. Krimm and J. E. Mark, Proc. Nat. Acad. Sci. U.S., 60, 1122 (1968).

4. T. Ooi, R. A. Scott, G. Vanderkoi, and H. A. Scheraga, J. Chem. P'hys., 46, 4410 (1967)

5. R. F. Epand and H. A. Scheraga, Biopolymers, 6, 1383 (1968).

S. KRIMM*

J. E. MARK $\dagger$

M. LOIS TIFFANY*

The University of Michigan

Ann Arbor, Michigan 48104

* Department of Physics and Biophysics Research Division

$\dagger$ Department of Chemistry 